\title{
Development of a Testbed for Robotic Neuromuscular Controllers
}

\author{
Alexander Schepelmann, Michael D. Taylor, Hartmut Geyer \\ The Robotics Institute \\ Carnegie Mellon University \\ Pittsburgh, Pennsylvania 15213 \\ Email: aschepelmann@cmu.edu,mdtaylor@cmu.edu,hgeyer@cs.cmu.edu
}

\begin{abstract}
Current control approaches to robotic legged locomotion rely on centralized planning and tracking or motion pattern matching. Central control is not available to robotic assistive devices that integrate with humans, and matching predefined patterns severely limits user dexterity. By contrast, biological systems show substantial legged dexterity even when their central nervous system is severed from their spinal cord, indicating that neuromuscular feedback controls can be harnessed to encode stability, adaptability, and maneuverability into legged systems. Here we present the initial steps to develop a robotic gait testbed that can implement and verify neuromuscular controls for robotic assistive devices. The initial stage consists of an antagonistically actuated two segment leg with a floating compliant joint. We detail its electromechanical design and low level, velocity-based torque control. Additionally, we present experiments that test the leg's performance during human-like high fidelity motions. The results show that the robot can track fast motions corresponding to $87 \%$ of the maximum performance limit of human muscle. The experiments also reveal limitations of our current implementation and we discuss solutions to overcoming them.
\end{abstract}

\section{INTRODUCTION}

Current approaches to leg control in locomotion either use centralized planning and tracking or mimic predefined joint motion patterns extracted from normal human gait. The first approach is used in humanoids including Honda's ASIMO and AIST's HRP-4 [11][18][27], but cannot be applied to robotic assistance wherein the central human user's state is unknown. As a result, the second approach prevails in rehabilitation robotics [17][2][12][13]. For instance, exoskeletons developed for paralyzed patients enforce a limited set of pre-defined motion patterns of normal human gait [21][1], which severely constrains their functional dexterity. In part, this problem can be overcome by combining motion libraries and pattern recognition, as Sup et al. [30] demonstrated with a powered legged prosthesis for speed and slope adaptation. However, control strategies that generate the stability, maneuverability, and adaptability needed for truly high mobility have not been identified with this approach.

We seek to develop an alternative control approach to powered legged systems that builds on decentralized neuromuscular control strategies of human locomotion. Animal and human legs possess remarkable autonomy in behavior and control. For example, decerebrate cats and rats have no brain control over their legs, yet they seamlessly adapt to different locomotion speeds on a treadmill and autonomously transition between gaits [28][4][20]. Similar neuro-scientific experiments reveal that, in biological systems, dexterous performance of segmented legs is realized to a large extent by local feedback controls that bypass central processing, and by biomechanical designs that hardcode functional leg responses [5] [24][14][7]. Recently, neuromuscular models of human locomotion were developed, which are controlled by autonomous local feedbacks without central planning, yet adapt to their environment and show substantial robustness of locomotion[8][29]. Eilenberg et al. [6] have implemented part of this feedback control in a powered ankle-foot prosthesis, resulting in a system that adapts to the environment without requiring explicit terrain sensing.

To generalize this approach to segmented powered legs, we here present our initial steps of developing a robotic gait testbed that can implement and test neuromuscular controllers for robotic assistive devices. The testbed currently consists of a half-human sized, two segment leg with two antagonistic actuators and a compliant floating joint. We detail the electromechanical design and control of this robotic neuromuscular leg (RNL) (sect. II and III), and present and discuss experimental results that test the performance of the leg during human-like, high-fidelity motions (sect. IV and V.

\section{EleCtromeChaniCAL DESIGN}

RNL is a half-human sized, two segmented, antagonistically actuated robotic leg with joint compliance (Fig. 1). The electromechanical design of RNL is driven by three themes: dynamic similarity, antagonistic actuation, and leg compliance.

\section{A. Dynamic Similarity}

We aim to build a testbed that matches human leg performance to develop neuromuscular controllers for powered segmented legs. For cost and safety considerations we build a robotic leg that is half the size and a quarter of the weight of a human leg. To ensure that the dynamic behavior of our robot matches human legs, we use dynamic scaling. Dynamic scaling uses fundamental physical variables to define relationships between a system's quantities at different scales. This approach was formalized by Buckingham [3] and is often applied in aerospace and fluid engineering applications. In mechanical systems, fundamental units are mass, length, and time. The robot's mass and length targets define these scaling factors 
(a)

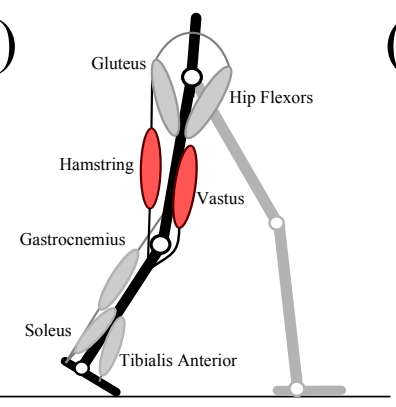

(b)

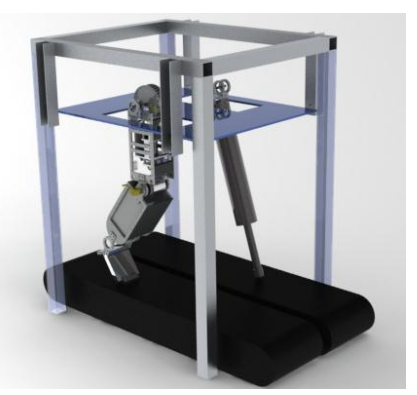

(c)

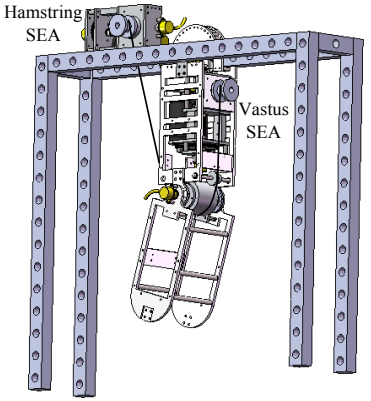

(d)

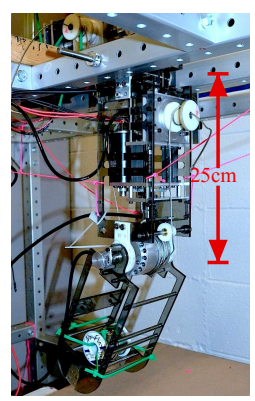

Fig. 1: Robotic testbed development. (a) Major human leg muscles with monoarticular knee extensor and biarticular knee flexor highlighted. (b) Testbed concept. (c) CAD assembly of initial stage. (d) Robotic implementation. For experiments, thigh was rigidly coupled to mounting cage.

\begin{tabular}{rccc} 
& Human & RNL & Scaling Factor \\
\cline { 2 - 4 } Leg Length $(\mathrm{m})$ & 1 & 0.5 & $1 / 2$ \\
Thigh Length $(\mathrm{m})$ & 0.46 & 0.23 & $1 / 2$ \\
Shank Length $(\mathrm{m})$ & 0.54 & 0.27 & $1 / 2$ \\
Knee Radius $(\mathrm{m})$ & 0.06 & 0.03 & $1 / 2$ \\
Total Mass $(\mathrm{kg})$ & 80 & 20 & $1 / 4$ \\
Thigh Mass $(\mathrm{kg})$ & 8 & 2 & $1 / 4$ \\
Shank Mass $(\mathrm{kg})$ & 3.7 & 0.9 & $1 / 4$ \\
Foot Mass $(\mathrm{kg})$ & 1.2 & 0.3 & $1 / 4$ \\
Vas. Max Force $(\mathrm{N})$ & 6000 & 1500 & $1 / 4$ \\
Vas. Max Vel $(\mathrm{m} / \mathrm{s})$ & 0.96 & 0.68 & $1 / 4$ \\
Ham. Max Force $(\mathrm{N})$ & 3000 & 750 & $1 / 4$ \\
Ham. Max Vel $(\mathrm{m} / \mathrm{s})$ & 1.2 & 0.84 & $\sqrt{2} / 2$ \\
Max Joint Torque $(\mathrm{Nm})$ & 368 & 45 & $1 / 8$ \\
Max Joint Vel $(\mathrm{rpm})$ & 153 & 217 & $\sqrt{2}$
\end{tabular}

TABLE I: Mechanical performance goals. Human mechanical properties dynamically scaled to RNL dimensions. Human data taken from Winter [32].

as $m_{\text {robot }} / m_{\text {human }}=1 / 4$ and $l_{\text {robot }} / l_{\text {human }}=1 / 2$, respectively. Since the robot is exposed to the same gravitational field as humans, the relationship $g_{\text {robot }}=g_{\text {human }}$ must hold, resulting in a time scale of $t_{\text {robot }} / t_{\text {human }}=\sqrt{1 / 2}$. Given these fundamental unit scales, force, torque, and velocity scale as $F_{\text {robot }} / F_{\text {human }}=1 / 4, \tau_{\text {robot }} / \tau$ human $=1 / 8$, and $v_{\text {robot }} / v_{\text {human }}=\sqrt{2} / 2$.

The performance envelope of human leg actuation is defined by the maximum contraction velocity and maximum isometric force that muscles can develop. We dynamically scale these two parameters to identify the equivalent actuator no load speed and torque requirements of RNL. The largest human leg muscle is the vastus, a knee extensor located in the front of the thigh (Fig. 17). The dominating muscle used in knee flexion is the hamstring, whose performance requirements are lower than those of the vastus. (Tab. 【).

\section{B. Antagonistic Actuation}

RNL uses antagonistic actuators, realized by cable driven series elastic actuators (SEAs), which simultaneously meets several design requirements: the ability to actively command zero joint torque to allow passive dynamics, satisfying human mass distributions, and actuation across floating joints.

SEAs were originally developed by Pratt and Williamson [25] and are common in bipedal robots such as Spring Flamingo developed by Pratt and Pratt [26] and M2 developed by Paluska [22]. SEAs are characterized by a compliant element between motor and load. This element decouples load and motor inertia, which enables precise torque control with highly geared motors (at the expense of system bandwidth), including zero torque. SEAs have been combined with cable drives in legged systems [10]. Using nondirect elements in the drivetrain, like cable, belt, and chain drives, allows actuators to be located away from the joint, ideal for realizing human-like segment mass distributions. Unlike belt and chain drives, cable drives can also go slack like human muscles, which allows them to act across compliant segments.

The drivetrain of RNL's SEAs is shown in Fig. 2p. With a later technology transfer to prosthetic and orthotic devices in mind, we limit ourselves to electric DC motors, focusing on the Maxon RE line. Several motor configurations match our design targets (Tab. III). The optimal combination of low weight, low rotor inertia, torque, and speed is reached by four mechanically coupled RE 30 motors. However, we instead opt for two mechanically coupled RE 40 motors to limit actuator complexity, at the expense of increased rotor inertia. For compactness, we incorporate a custom three stage (a)

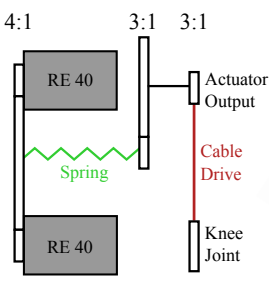

(b)

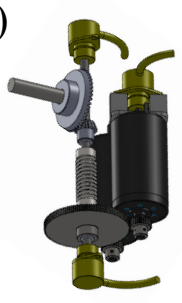

(c)

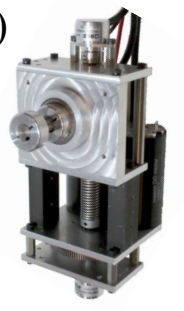

Fig. 2: RNL SEAs. (a) Drivetrain schematic (b) Drivetrain assembly (c) Prototype 
4X RE $30 \quad 2 \times$ RE 40

$\begin{array}{rcc}\text { Gear Ratio } & 40 & 36 \\ \text { Total Weight }(\mathrm{g}) & 952 & 960 \\ \text { Rotor Inertia }\left(\mathrm{kgm}^{2}\right) & 0.0221 & 0.03 \\ \text { Nominal Torque }(\mathrm{Nm}) & 14.1 & 13.2 \\ \text { Stall Torque }(\mathrm{Nm}) & 163 & 180 \\ \text { No Load Speed }(\mathrm{rpm}) & 212 & 211 \\ \text { Nominal Speed }(\mathrm{rpm}) & 194 & 194\end{array}$

TABLE II: SEA motor configurations. Optimal configuration is comprised of four RE 30s. Two RE 40s meet the same performance criteria with lower mechanical complexity, at the expense of increased rotor inertia.

(a)

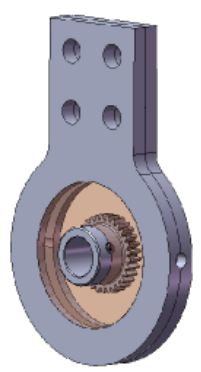

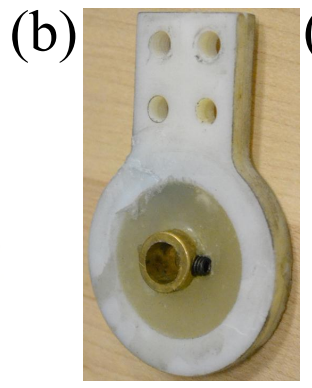

Fig. 3: Floating compliant knee joint. (a) Concept (b) Prototype (c) Close-up of implementation

drivetrain into our actuation system (Fig. 2a). The first stage mechanically couples the motors with a 4:1 reduction; the second stage orients the output shaft's axis of rotation with a 3:1 reduction. We locate a rotary spring coupling between these stages. Currently, we use an off-the-shelf spring coupler (Stock Drive Products) with a stiffness of $1.75 \mathrm{Nm} / \mathrm{rad}$ and a maximum torque rating of $1.4 \mathrm{Nm}$. An additional 3:1 reduction is located between the SEA output shaft and joint, connected via cable drive, for a total 36:1 gear reduction in our drivetrain. The gear ratio of the external stage can be modified, which allows us to use the same SEA for muscles with different properties. We realize force measurements via two absolute rotary encoders (RM22B: 9bit, magnetic, analogue encoders; Renishaw PLC) located on either side of the spring. Additionally, an incremental encoder on the shaft of one RE 40 measures motor velocity (RM22I: 9bit, magnetic, digital encoder; Renishaw PLC). One motor controller (Solo Whistle, Elmo MC) supplies the same current to both RE 40s. Currently RNL uses two SEAs. One is located in the robot's thigh, simulating the vastus muscle; the other is located on the mounting frame, simulating the biarticular hamstring muscle (Fig. 17).

\section{Leg Compliance}

Humans are not rigidly coupled kinematic chains, possessing interjoint cartilage and soft tissue around bones. To capture this aspect in RNL, we incorporate a floating joint design into the robot (Fig. 3). The floating joint design connects the robot's thigh and shank. The joint is composed of two rapid prototyped clamping plates (VeroWhitePlus, Objet Ltd.), which hold the compliant element. The compliant element is made from a two-part PMC-744 urethane rubber mix (Smoothon Inc.), which is cast into the clamping plates. A compliance retainer, constructed from a brass spur gear, couples the joint's shaft and compliant element, and defines the floating center of rotation of the knee joint's axis. The retainer's set screw rigidly couples the thigh and joint shaft. This design restricts rotational motion to only occur in the robot's shank. An RM22B encoder head is mounted on the joint shaft. The corresponding encoder body is located on the shank. Relative motion between the encoder head and body measures knee position during leg movement.

\section{Velocity-BASEd SEA CONTROL}

Our actuators generate desired torques using a velocitybased SEA control scheme [31]. Pratt and Williamson [25] originally approached SEA control from a torque-based perspective. Recently, an alternative SEA controller was proposed by Wyeth [33], which modulates load torque, $\tau_{l}$, using motor velocity, $\omega_{m}$, as a control target instead of motor torque,

$$
\omega_{m}=\tau_{l}\left(\frac{1}{J_{l} s}+\frac{s}{k_{s}}\right)
$$

where $J_{l}$ is the load inertia and $k_{s}$ is the stiffness of the compliant element. This approach is advantageous because losses due to gear dynamics between the motor and spring do not need to be considered. Since motor velocity corresponds exactly to velocity at the drivetrain output, the velocity loop automatically compensates for losses without additional tuning of the outer control loop. In addition, the controller's inverse dynamics terms (Fig. 4) only require the first derivative of motor position, which leads to increased system bandwidth, since low-pass filters with higher cutoff frequencies can be used.

Wyeth [33]'s formulation of velocity-based SEA control (eq. 11) requires load inertia to be known. For legged systems, it is not clear what the load inertia is, as load dynamics constantly change due to joint position and gait phase. However, knowledge of load inertia is not necessary in the formulation of a velocity-based SEA controller. Starting with Pratt and Williamson [25]'s derivation, torque exerted by a spring due to angular deflection is given as

$$
\tau_{l}=-k_{s}\left(\theta_{l}-\theta_{m}\right)
$$

where $\tau_{l}$ is the torque applied to the load, $k_{s}$ is the spring stiffness, and $\left(\theta_{l}-\theta_{m}\right)$ defines the deflection of the spring between the load and motor side. To formulate torque control as velocity-based control, we write motor position as a function of motor velocity $\theta_{m}=\omega_{m} / s$, and resolve eq. 2 to

$$
\omega_{m}=\tau_{l} / k_{s} s+\theta_{l} s
$$

Here $J_{l}$ does not need to be known. Fig. 4 shows a schematic implementation of this velocity control, in which eq. 3 has been implemented as feedforward compensation, $P(s)$ is PD feedback compensation for model uncertainty, and $C(s)$ represents the motor controller. 


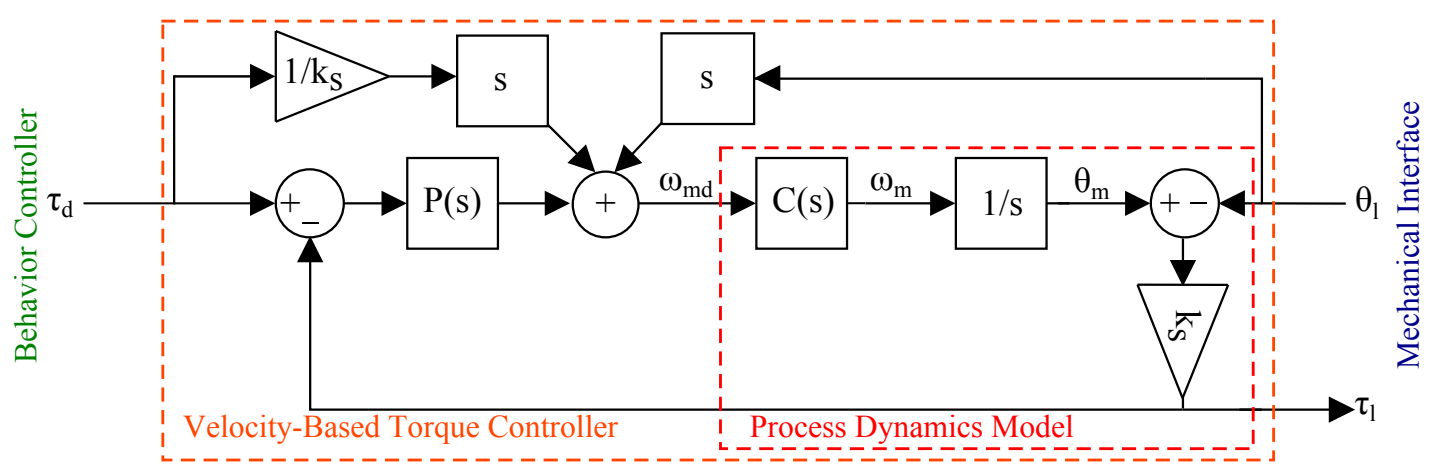

Fig. 4: Velocity-based SEA control system model.

(a)
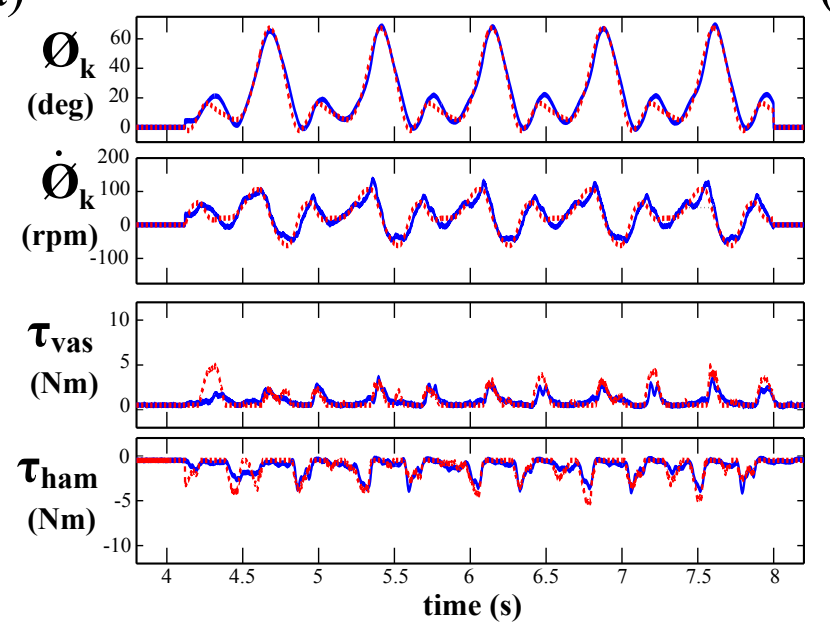

(c)

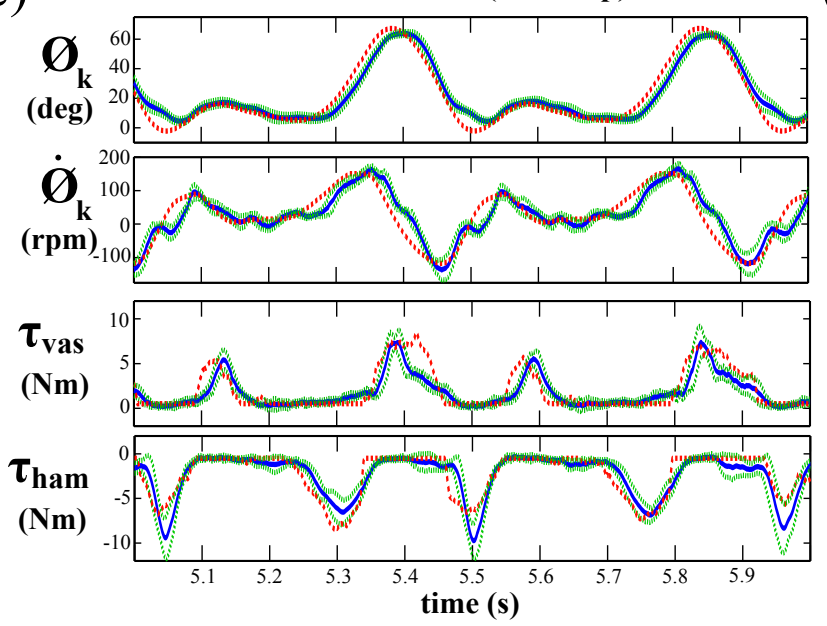

(b)
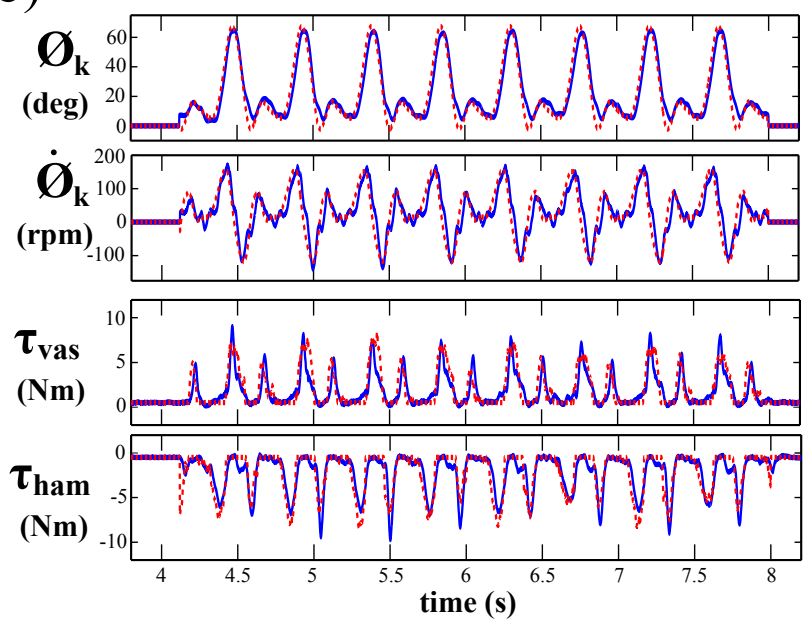

(d)

1.8x Nominal
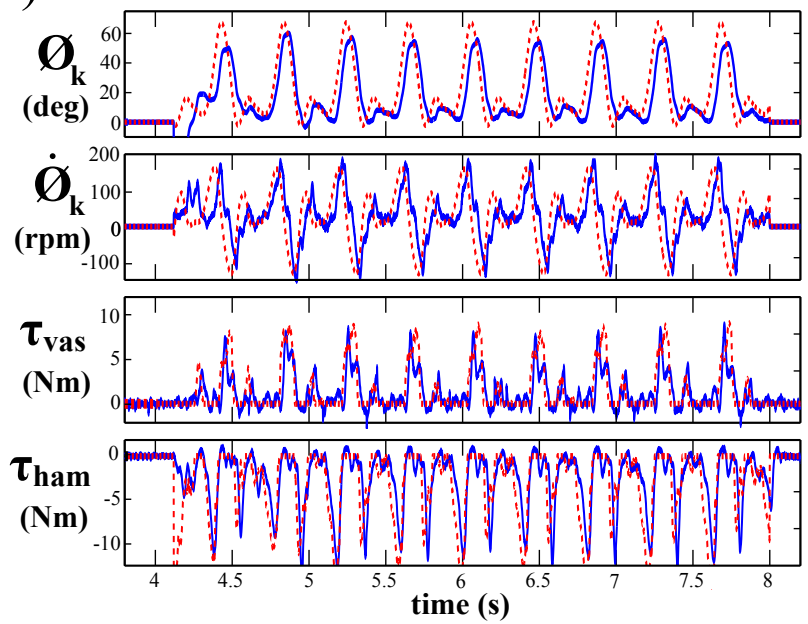

Fig. 5: High fidelity motion experiments. RNL knee position, velocity, and antagonistic actuator torques for walking trajectories corresponding to (a) 1.0x, (b,c) 1.6x, and (d) 1.8x nominal walking speed trajectories. In experiments, motion occurred between $4 s$ and $8 s$. Plots show mean \pm s.d. for 10 repetitions. Red lines: commanded trajectories. Blue lines: measured trajectories. Green lines: \pm s.d. of measured trajectories.

\section{EXPERIMENTS}

The electromechanical design of RNL includes custom built antagonistic actuators, an alternative velocity-based SEA controller, and a floating compliant knee joint. RNL is intended as platform to develop neuromuscular controllers that embed human-like performance. To verify if our design and control 
(a)
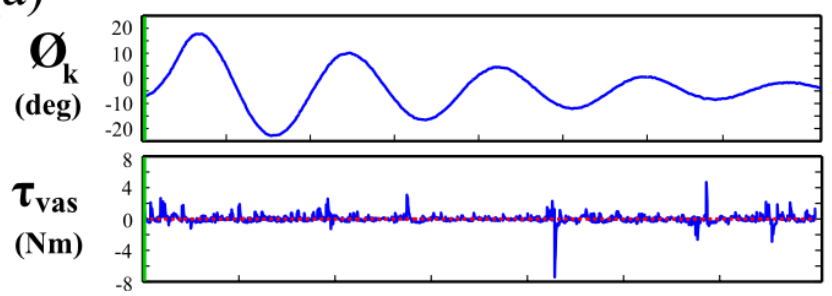

$\tau_{\text {ham }}$

(Nm)

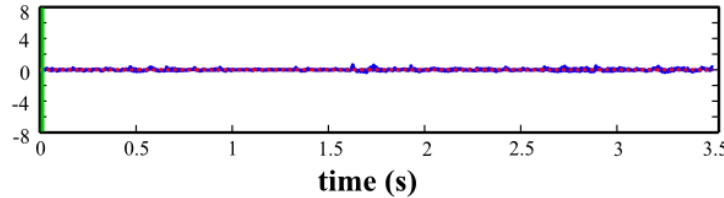

(c)

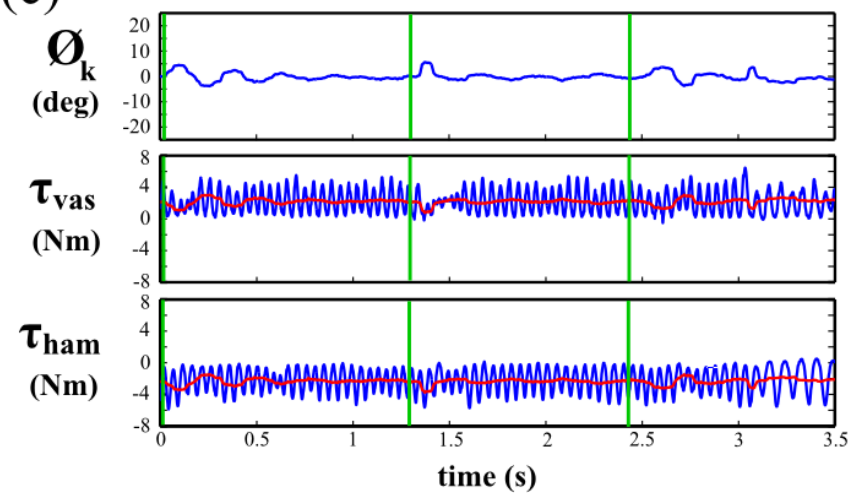

(b)

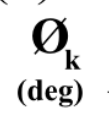

$\tau_{\text {vas }}$
(Nm)

$\tau_{\text {ham }}$

(Nm)
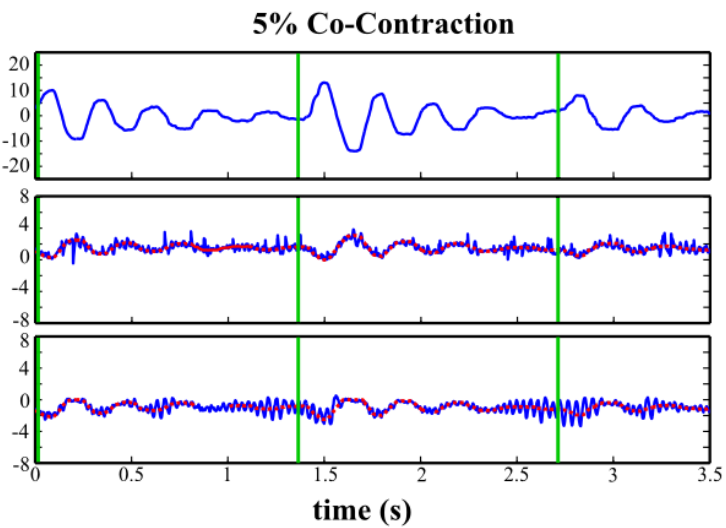

$15 \%$ Co-Contraction

(d)
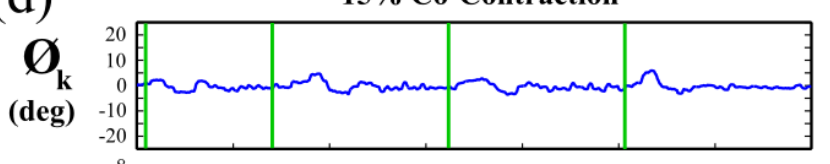

$\tau_{\text {vas }}$

(Nm)

$\tau_{\text {ham }}$

(Nm)

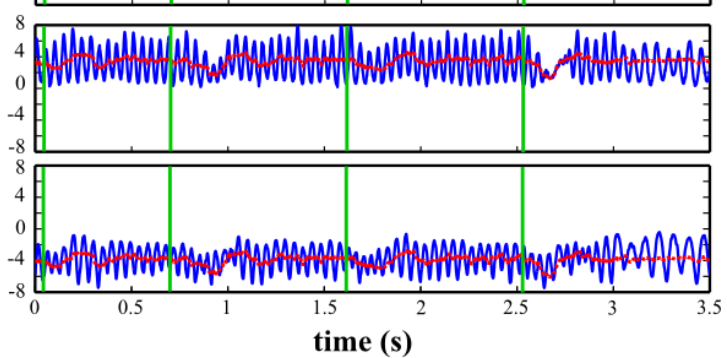

Fig. 6: RNL knee position and antagonistic actuator torque during co-contraction experiments for (a) $0 \%$ (b) $5 \%$ (c) $10 \%$ (d) 15\%. Red lines: commanded trajectory. Blue lines: measured trajectory. Green lines: External impulse disturbances to instigate shank oscillation.

implementations meet this performance, we devise two experiments. The first experiment tests if the robot can generate fast, high fidelity motions that characterize human locomotion. The second experiment targets the ability to execute antagonistic co-contraction often seen in neuromuscular control.

Since RNL currently only has actuators simulating the hamstring and vastus, we focus on the robot's knee for this evaluation. To simulate the inertial effects of a foot segment, we attach a weight to the bottom of RNL's shank, increasing its total mass to $1.1 \mathrm{~kg}$. The resulting mechanical properties of the shank segment are $l_{\text {com }}=0.107 \mathrm{~m}$ and $J_{s}=0.005 \mathrm{kgm}^{2}$, where $l_{\text {com }}$ is the distance of the shank's center of mass position from the knee pivot and $J_{s}$ is the inertia.

\section{A. High Fidelity Motion Experiments}

We evaluate RNL's motion fidelity using dynamically scaled human joint trajectories. We first numerically differentiate knee angular position data, $\phi_{k}^{r e f}$, tabulated in Winter [32] to generate reference trajectories for joint velocity, $\dot{\phi}_{k}^{\text {ref }}$, and acceleration, $\ddot{\phi}_{k}^{r e f}$, observed in human walking. The trajectories are median filtered (filter order $=10$ ) to eliminate artifacts resulting from the differentiation. Next, we use dimensional scaling to adapt the trajectories to RNL's scale. The resulting joint trajectories correspond to normal human walking speed (1.0x nominal trajectory). We scale these trajectories to range between 1.0x and 2.0x speed of the nominal trajectory, the latter of which reaches maximum knee joint velocity (Tab. (1).

With these references, we implement a tracking control that outputs desired actuator torques. The controller includes feedforward torque trajectories, $J_{s} \ddot{\phi}_{k}^{r e f}$, gravity compensation, and PD feedback compensation, $k_{p}\left(\phi_{k}^{r e f}-\phi_{k}\right)+k_{d}\left(\dot{\phi}_{k}^{r e f}-\dot{\phi}_{k}\right)$, where $k_{p}=15$ and $k_{d}=0.01 k_{p}$ are the position and velocity feedback gains. The gains are kept constant throughout all experiments. $\phi_{k}$ and $\dot{\phi}_{k}$ are measured by the absolute encoder on RNL's knee joint. The four components are summed to generate net joint torque. The resulting actuator flexion and extension torques are commanded to the corresponding SEA via the velocity-based control scheme described in section III To avoid cable slack, each SEA applies a minimum $0.5 \mathrm{Nm}$ torque to the joint at all times. Fig. 5 summarizes the results of the motion experiments. Tabs. [II] and IV list the mean crosscorrelation coefficients and signal time delays over the trials for all traces. We observe that the executed joint position and velocity trajectories closely follow the desired trajectories for trials up to $1.6 \mathrm{x}$, with position and velocity correlation coefficients greater than 0.90 and 0.80 , respectively. The largest differences between desired and commanded position occur during periods of knee extension at 1.6x, with a maximum error of $7.3^{\circ}$. Velocity shows a similar tracking quality. Top speeds achieved 160rpm in 1.6x trials. In addition, we observe 


\begin{tabular}{ccccc} 
& $R_{\theta_{k}}$ & $R_{\dot{\theta}_{k}}$ & $R_{\tau_{\text {vas }}}$ & $R_{\tau_{\text {ham }}}$ \\
\cline { 2 - 5 } $1.0 x$ & $0.97 \pm 0.01$ & $0.87 \pm 0.04$ & $0.71 \pm 0.06$ & $0.90 \pm 0.02$ \\
$1.2 x$ & $0.97 \pm 0.01$ & $0.88 \pm 0.02$ & $0.67 \pm 0.04$ & $0.90 \pm 0.02$ \\
$1.4 x$ & $0.94 \pm 0.02$ & $0.82 \pm 0.04$ & $0.80 \pm 0.02$ & $0.82 \pm 0.05$ \\
$1.6 x$ & $0.95 \pm 0.02$ & $0.85 \pm 0.02$ & $0.80 \pm 0.01$ & $0.80 \pm 0.03$ \\
$1.8 x$ & $0.80 \pm 0.06$ & $0.64 \pm 0.08$ & $0.77 \pm 0.04$ & $0.62 \pm 0.04$
\end{tabular}

TABLE III: Mean correlation coefficients $(R)$ for walking trials. $n=10$ for all speeds.

\begin{tabular}{ccccc} 
& $t_{\theta_{k}}$ & $t_{\dot{\theta}_{k}}$ & $t_{\tau_{\text {vas }}}$ & $t_{\tau_{\text {ham }}}$ \\
\cline { 2 - 5 } $1.0 x$ & $9.5 \pm 1.5$ & $18.4 \pm 2.6$ & $13.1 \pm 1.7$ & $4.8 \pm 0.9$ \\
$1.2 x$ & $7.2 \pm 0.9$ & $11 \pm 0.7$ & $10.3 \pm 1.2$ & $5.1 \pm 0.7$ \\
$1.4 x$ & $15.9 \pm 1.4$ & $19.5 \pm 1.8$ & $9.1 \pm 1.0$ & $9.0 \pm 1.6$ \\
$1.6 x$ & $12.3 \pm 2.4$ & $15.2 \pm 2.3$ & $10.7 \pm 0.8$ & $9.3 \pm 1.7$ \\
$1.8 x$ & $25.0 \pm 3.9$ & $25.2 \pm 4.4$ & $9.5 \pm 2.1$ & $14.9 \pm 0.9$
\end{tabular}

TABLE IV: Mean time delays $(t)$ in $m s$ for walking trials. $n=10$ for all speeds.

a high degree of repeatability throughout all trials, with a maximum standard deviation of $7.4^{\circ}$ and $44 \mathrm{rpm}$ from the mean values over all trial 1 The results indicate that humanlike segment motions can be reliably and accurately replicated for speeds up to $160 \mathrm{rpm}$.

At higher speed trials, the quality of position and velocity tracking declines (Tabs. III and IVP. Nevertheless, velocity targets were still reached with top speeds of at least $190 \mathrm{rpm}$ (Fig. 5d), or about $87 \%$ of our desired performance goals outlined in Tab. II We could not test higher speeds, because the compliance retainer in the knee joint failed during the high speed trials.

\section{B. Co-Contraction Experiments}

Antagonistic muscle co-contraction is characterized by equal pre-load torques of the muscles, $\tau_{P L}$, and an equivalent rotational joint stiffness, $k_{k n e e}$. The corresponding desired SEA torques are given by

$$
\tau_{\text {vas } / \text { ham }}=\tau_{P L} \pm k_{\text {knee }}\left(\phi_{k}-\phi_{0}\right)
$$

where $\phi_{0}$ is the joint reference position. To estimate appropriate values for $\tau_{P L}$ and $k_{k n e e}$, we use a Hill-type muscle model as described in Geyer et al. [9]. In these models, the muscle force, $F$, is given by

$$
F=A f_{l} f_{v} F_{\max }^{i s o}
$$

where $A$ is percentage of muscle activation, $F_{i s o}^{\max }$ is the muscle's maximum isometric force, and $f_{l}$ and $f_{v}$ are the force-length and force-velocity relationships. Combined with the knee joint radius, the muscle force defines $\tau_{P L}$. For calculating the local stiffness, we neglect modulations by $f_{v}$ and model $f_{l}$ as

$$
f_{l}=\exp \left[\ln (0.05)\left|\frac{l_{C E}-l_{o p t}}{0.056 l_{\text {opt }}}\right|^{3}\right]
$$

\footnotetext{
${ }^{1}$ The first leg swing was not included in this calculation, as impulse accelerations from rest is not representative of system dynamics.
}

\begin{tabular}{cccc}
$A(\%)$ & $k_{\text {muscle }}(\mathrm{N} / \mathrm{m})$ & $\tau_{P L}(\mathrm{Nm})$ & $k_{\text {knee }}(\mathrm{Nm} / \mathrm{rad})$ \\
\hline & 0 & 0 & 0 \\
5 & 7310 & 1.22 & 6.59 \\
10 & 14640 & 2.44 & 13.17 \\
15 & 21950 & 3.66 & 19.79
\end{tabular}

TABLE V: Equivalent stiffnesses \& SEA pre-load torques at different muscle activation levels. Values calculated at $l_{C E}=$ $1 / 2 l_{\text {opt }}$.

\begin{tabular}{rccc} 
& $\mathbf{0 \%}$ & $\mathbf{5 \%}$ & $\mathbf{1 0 \%}$ \\
\cline { 2 - 4 }$T$ Simulation $(s)$ & 0.78 & 0.30 & 0.22 \\
$T$ RNL $(s)$ & $0.75 \pm 0.04$ & $0.25 \pm 0.01$ & $0.25 \pm 0.02$
\end{tabular}

TABLE VI: Period of oscillation at different levels of cocontraction.

where $l_{C E}$ is the length of the muscle's contractile element and $l_{\text {opt }}$ is its optimal length. The resulting muscle stiffness is

$$
k_{\text {muscle }}=A \frac{d f_{l}}{d l_{C E}} F_{\text {max }}^{i s o} \text {. }
$$

Using scaled values of the vastus complex $\left(l_{\text {opt }}=0.04\right.$, $w=0.056$ ), we calculate $k_{\text {muscle }}, \tau_{P L}$, and $k_{\text {knee }}$, for four levels of $A$, ranging from $0 \%$ to $15 \%$ muscle activation. (Tab. V.

If RNL can successfully execute co-contraction, the motion resulting from the torques described by eq. 4 matches the motion of a physical driven pendulum whose center of mass and inertia properties correspond to those of the shank. We test the quality of co-contraction control by deflecting the shank from its rest position $\phi_{0}=0$ and comparing the resulting behavior to that of a simulated, equivalent driven physical pendulum.

Fig. 6 shows the behavior of RNL during the co-contraction experiments. Fig. 6a shows the observed motion and torques for $0 \%$ co-contraction, which evaluates the quality of RNL's zero torque control. The shank follows the motion of a damped pendulum with a period $T$ that matches the simulated period (Tab. VI). For $0 \%$ co-contraction, the desired $\tau_{\text {vas } / \text { ham }}$ is zero. The measured torques track the commanded torque within torque resolution limits of the SEAs. $( \pm 0.1 \mathrm{Nm}$ joint torque resolution for SEA spring stiffness of $1.75 \mathrm{Nm}$ and 9 bit encoders.) Higher levels of co-contraction are possible (Fig. 6b-d), but the commanded torques are not well tracked, as large oscillations result from the antagonistic actuators counteracting each other with large pre-load torques. Because the oscillations exceed the maximum torque rating of our spring at approximately $15 \%$ co-contraction, we cannot command higher levels of co-contraction. This shortcoming of our current implementation of antagonistic control is visible in the position trajectories as well.

\section{Discussion \& CONClusion}

Our goal is to develop neuromuscular controllers for powered segmented legs. To realize this goal, we presented the 
initial design and validation stage of a robotic test platform targeting human-like performance. The platform currently consists of RNL, a half-human sized, two segment robotic leg with a floating compliant knee joint (Fig. 11). The electromechanical design of the leg meets the physical weightsize properties and actuation performance defined by human physiology. To meet these criteria, we developed a modular, compact SEA (Fig. 2) that matches the performance of the vastii, the largest muscle group in human legs. In addition, we formulated an SEA control that takes advantage of velocitybased torque control without having to know load inertia (Fig. 4).

In two experiments, we tested if our design and control implementation can deliver fast motions that characterize human locomotion and can generate antagonistic co-contraction seen in neuromuscular systems. The experimental results show that we can reliably generate human-like leg motions with high positional accuracy for joint speeds up to 160rpm and with lesser positional accuracy for joint speeds up to $190 \mathrm{rpm}$ (Fig. 5), approximately $90 \%$ of the maximum designed for joint velocity (Tab. I). The failure of the knee joint's clamping plates during the high speed experiments indicates that the prototyped plates will need to be replaced by stronger material versions to achieve these maximum speeds. On the other hand, the compliant part of the floating joint did not show any wear throughout the experiments, suggesting that its design works very well. The co-contraction experiments revealed that the antagonistic actuators can command zero torque within the torque resolution limits of the SEAs, enabling near passive motions at the joint level (Fig. 6a). Higher levels of cocontraction up to $15 \%$ of human muscle activations were possible, but produced oscillations in the SEA torque patterns which limited the performance of co-contraction control.

The experiments revealed two shortcomings of our current implementation, which we are working on overcoming. First, the actuators' bandwidth limitation creates about $25 \mathrm{~ms}$ of time delay during high speed position and velocity tracking. Scaled to human dimensions, this delay would be about $35 \mathrm{~ms}$, which is similar to the feedback delays observed in humans. However, the SEA delay will increase substantially with larger actuation levels necessary for stance motions. To diminish the delay, we are looking to replace the linear rotary spring in the actuators with a nonlinear stiffening spring. Stiffening springs in SEAs enable high precision zero torque control with high bandwidth responses at large commanded torques. The advantages of nonlinear springs to series elastic actuation are widely recognized (Pratt and Williamson [25], Hurst et al. [15], Migliore et al. [19]), although a systematic method for developing compact springs with custom force deflection profiles has not been proposed. In addition, a custom nonlinear spring coupler will also allow us to overcome the current torque limit of $12.6 \mathrm{Nm}$ at the output that is defined by the maximum torque capacity of the off-the-shelf springs in the SEAs.

The second shortcoming is the $15 \%$ limit on co-contraction control. The impact of this limit on implementing neuromus- cular control strategies is unclear. While humans can reach up to $30 \%$ of antagonistic co-contraction at the leg joints [23], these levels are observed during the stance phase in which the legs are loaded by body weight, which is about 17 times larger than the weight of the shank. In the experiments, by contrast, the leg was unloaded and resistance to motion was entirely due to the mass properties of the shank. In particular, already at $5 \%$ co-contraction the peak torques of the actuators reached $3.2 \mathrm{Nm}$, which corresponds to the torque created by one body weight. Hurst et al. [16] suggest that damping elements parallel to the SEA spring may attenuate oscillations when load inertia is low. We plan to test if adding such elements into the SEA drivetrain will improve the quality of co-contraction control.

The presented robot validates our initial design and control implementation, but does not represent a full neuromuscular leg. We are currently expanding RNL into a multi-degree of freedom leg with hip, knee, and ankle joints as well as an adaptive, compliant foot. This robot will incorporate 7 actuators based on our presented SEA design and control implementation. These actuators represent the major monoand bi-articular muscles that propel human legs during walking and running. The actuators will be controlled by reflexive neuromuscular models [8] [29], which will generate desired torques for each SEA unit ( $\tau_{d}$ in Fig. (4). With this robot, the immediate goal will be to realize reflexive leg controls during swing, crucial for autonomous balance recovery in amputee locomotion. Ultimately, we expect this work to result in a generalized gait testbed to develop and test neuromuscular controllers for multi-articulated powered robotic limbs for rehabilitation and humanoid robotics.

\section{ACKNOWLEDGMENTS}

This work is supported by the Richard King Mellon Foundation. A.S. is supported by the National Science Foundation Graduate Research Fellowship Program. The authors wish to thank Larry Hayhurst and David Matten for their help with robot construction. We also wish to thank Daniel Häufle for his help with hardware communication.

\section{REFERENCES}

[1] S.K. Agrawal, S.K. Banala, K. Mankala, V. Sangwan, J.P. Scholz, V. Krishnamoorthy, and W.L. Hsu. Exoskeletons for gait assistance and training of the motor impaired. Proc IEEE Int Conf Rehab Robo, pages 1108-1113, 2007.

[2] H.K. Au, H. Herr, J. Weber, and E.C. MartinezVillalpando. Powered ankle-foot prosthesis for the improvement of amputee ambulation. Conf Proc IEEE Eng Med Biol Soc, pages 3020-3026, 2007.

[3] E. Buckingham. On physically similar systems; illustrations of the use of dimensional equations. Phys Rev, 4: 345-376, 1914.

[4] G. Courtine, Y. Gerasimenko, R. van den Brand, A. Yew, P. Musiekno, H. Zhong, B. Song, Y. Ao, R.M. Ichiyama, I. Lavrov, R.R. Roy, M.V. Sofroniew, and V.R. Edgerton. 
Transformation of nonfunctional spinal circuits inot functional states after the loss of brain input. Nat Neurosci, 12(10):1333-1342, 2009.

[5] V. Dietz. Proprioception and locomotor disorders. Nat Rev Neurosci, 3(10):781-790, 2002.

[6] M.F. Eilenberg, H. Geyer, and H. Herr. Control of a powered ankle-foot prosthesis based on a neuromuscular model. IEEE Trans Neural Sys Rehab Eng, 18(2):164$173,2010$.

[7] Y. Gerasimenko, R.R. Roy, and V.R. Edgerton. Epidural stimulation: comparison of the spinal circuits that generate and control locomotion in rats, cats and humans. Experimental Neurology, 209(2):417-425, 2008.

[8] H. Geyer and H. Herr. A muscle-reflex model that encodes principles of legged mechanics produces human walking dynamics and muscle activities. IEEE Trans Neural Sys Rehabil Eng, 2010.

[9] H. Geyer, Seyfarth A., and R. Blickhan. Positive force feedback in bouncing gaits? Proc R Soc B, 270:21732183, 2002.

[10] J.W. Grizzle, J. Hurst, B. Morris, H.W. Park, and K. Sreenath. Mabel, a new robotic bipedal walker and runner. American Control Conference, pages 2030-2036, 2009.

[11] M. Hirose and K. Ogawa. Honda humanoid robots development. Phil Trans $R$ Soc A, 365(1850):11-19, 2007.

[12] J. Hitt, A.M. Oymagil, T. Sugar, K. Hollander, Boehler A., and J. Fleeger. Dynamically controlled ankle-foot orthosis (dco) with regenerative kinematics: incrementally attaining user portability. Proc IEEE ICRA, pages 15411546, 2007.

[13] M.A. Holgate, A.W. Boehler, and T. Sugar. Control algorithms for ankle robots: a reflection on the state-of-theart and presentation of two novel algorithms. IEEE/RASEMBS Int Conf Biomed Rob and Biomech, pages 97-102, 2008.

[14] H. Hultborn and J.B. Nielsen. Spinal control of locomotion - from cat to man. Acta Physiologica, 189(2): 111-121, 2007.

[15] J.W. Hurst, J.E. Chestnutt, and A.A. Rizzi. An actuator with physically variable stiffness for highly dynamic legged locomotion. Proc IEEE ICRA, pages 4662-4667, 2004.

[16] J.W. Hurst, A.A. Rizzi, and D. Hobbelen. Series elastic actuation: Potential and pitfalls. Proc Int Conf. Climb Walk Robots, pages 1-6, 2004.

[17] R. Jimenez-Fabian and O. Verlinden. Review of control algorithms for robotic ankle systems in lower-limb orthoses, prostheses, and exoskeletons. Med Eng Phys, 34 (4):397-408, 2012.

[18] S. Kajita, T. Nagasaki, K. Kaneko, and H. Hirukawa. Zmp-based biped running control. IEEE Robotics and Automation Magazine, 14(2):63-72, 2007.

[19] S.A. Migliore, E.A. Brown, and S.P. DeWeerth. Novel nonlinear elastic actuators for passively controlling robotic joint compliance. J Mech Des, 129(4):406-412, 2007.

[20] P. Musienko, R. van den Brand, O. Maerzendorfer, R.R. Roy, Y. Gerasimenko, V.R. Edgerton, and G. Courtine. Controlling specific locomotor behaviors through multidimensional monoaminergic modulation of spinal circuitries. J Neurosci, 31(25):9264-9278, 2011.

[21] P.D. Neuhaus, J.H. Noorden, T.J. Craig, J. Kirschbaum, and J.E. Pratt. Design and evaluation of mina: A robotic orthosis for paraplegics. Proc IEEE ICORR, pages 1-8, 2011.

[22] D.J. Paluska. Design of a humanoid biped for walking research. MS Thesis, Massachusetts Institute of Technology, 2000.

[23] J. Perry and J.M. Burnfield. Gait analysis: Normal and pathological function. SLACK Incorporated, 2, 2010.

[24] E. Pierrot-Desseilligny and D. Burke. The circuitry of the human spinal cord: its role in motor control and movement disorders. Cambridge University Press, 2005.

[25] G.A. Pratt and M.M. Williamson. Series elastic actuators. Proc IEEE/RSJ IROS, 1:399-406, 1995.

[26] J.E. Pratt and G.A. Pratt. Intuitive control of a planar bipedal walking robot. Proc IEEE ICRA, 3:2014-2021, 1998.

[27] M. Raibert. Legged robots that balance. MIT Press, Cambridge, 1986.

[28] M.L. Shik, F.V. Severin, and G.N. Orlovski. Control of walking and running by means of electric stimulation of the midbrain. Biofizika, 11(4):659-666, 1966.

[29] S. Song and H. Geyer. Adaptation of local feedback control for large speed transitions in human-like walking. Proc IEEE ICRA, 2012.

[30] F. Sup, H.A. Varol, and M. Goldfarb. Upslope walking with a powered knee and ankle prosthesis: initial results with an amputee subject. IEEE Trans Neural Syst Rehavil Eng, 19(1):71-78, 2011.

[31] M.D. Taylor. A compact series elastic actuator for bipedal robots with human-like dynamic performance. MS Thesis, Carnegie Mellon University, 2011.

[32] D.A. Winter. Biomechanics and motor control of human movement. Wiley, (4), 2009.

[33] G. Wyeth. Control issues for velocity sourced series elastic actuators. Proc IEEE ICRA, 2006. 